\title{
STRINGS : A HAPTIC INTERFACE
}

Myrto Karanika

MFA Computational Studio Arts

Goldsmiths University of London

New Cross

London SE14 6NW

United Kingdom

myrtou@gmail.com

\begin{abstract}
This paper presents my attempt to design an installation that investigates the bi-directional relation of affect we share with our environment. The focus is on the design of an innovative fabric prototype that presents a range of textural qualities to trigger bodily expressions, and which is also capable of perceiving such expressions itself in a tangible manner without the use of distant modes of interactions such as sensors and motion-tracking systems.
\end{abstract}

\section{INTRODUCTION}

Investigating the bi-directional relation that we share with our surroundings, my work is narrowing down the focus on the relationships between space and bodily expression. My investigations are equally drawn from both space and the body, specifically looking into the ways in which the expressive body is affected (touched) by the space that surrounds it and vice versa.

In this paper, I will briefly present Strings, an installation that has emerged as a study on the design of a responsive haptic environment and the bi-directional relation of affect it shares with its users. My approach has been to create such an environment through the use of soft materials and soft technologies that attempt to evoke and capture bodily expressions in a tactile manner without the use of distant modes of interactions such as sensors or vision-based tracking systems. I have, for these purposes, designed an innovative fabric to be used as a spatial envelope, the weaving of which involves a combination of conductive and non-conductive threads. Within the created space, all the physical bodily gestures of the users upon the envelope are translated into virtual strings of topographical data that map each distinct gesture and translate it into sounds of stringed instruments within MAX/MSP. In this way an interrelation among gestural, computational, weaving and musical strings occurs that links the space with the users' senses of touch, vision and hearing. That sets the ground for a bi-directional relation of affect between the audio-haptic qualities of the space and the users' engagement with them.

The following section is prefaced by a short analysis on the concepts of hapticity and haptic space based on a selection of readings from architects and theoreticians. It continues with an overview of Strings and its placement within the fore-mentioned theoretical platform. The rest of the paper is divided into a number of subsections that focus on the textural and technical details of the installation. The last section is 
concerned with a reflection on the progress of the work so far, and with some thoughts on its future development.

\section{HAPTICITY, HAPTIC SPACE \& BODILY EXPRESSIONS}

In their Thousand Plateaus, Deleuze \& Guattari [1] note that haptic "may be as much visual or auditory as tactile", acknowledging within the term a sensory interrelation of the eye and the ear with the limbs. As a concept, therefore, haptic can be used to address our embodied experience of a space's textural qualities (weight, mass, density, pressure, humidity, temperature, presences, and resonances), as an experience that is simultaneously orchestrated by our vision, hearing and touch. However, haptic can also be extended to involve emotional connotations and to reflect affective response. The term has been repeatedly used likewise by theoreticians like Merleau-Ponty [2], Kant [3] and Paterson [4].
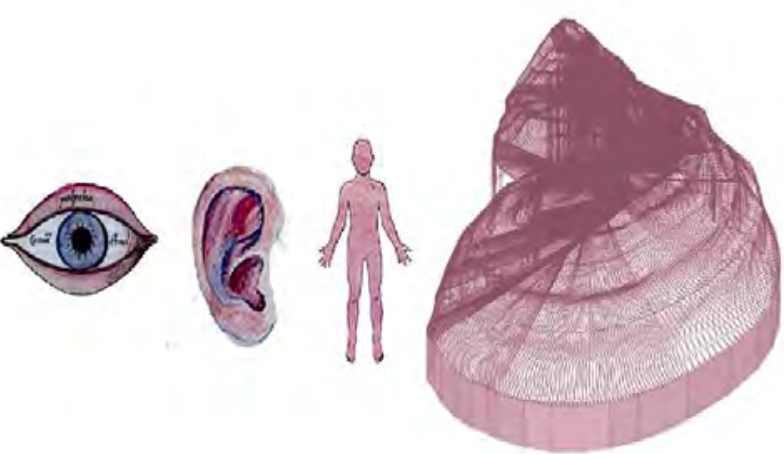

Figure 1. Hapticity: interrelation of the eye, the ear and the limbs.

Translating the words 'haptic', 'sense' and 'emotion' in Greek, my mother language, an interconnection lies within the three concepts: Haptic originates in the Greek word $\alpha \pi \tau$, which means something that can be touched or grasp-ed. Sense, translated as

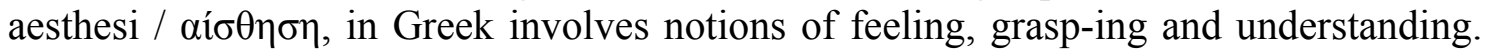
Consequently, the concept of 'grasp' is core in both aesthesi (sense) and haptic. Emotion on the other hand, translated in Greek as $\alpha i \sigma \theta \eta \mu \alpha$ / aesthema, shares the same root with aesthesi, as both derive from the word $\alpha \iota \sigma \theta \alpha$ 'vo $\mu \alpha \mathrm{l} /$ aesthanome, whose ambiguous meaning can be equally translated as 'I sense' or 'I feel'. Among these three words - haptic, sense, and emotion - there is an underlying relation that brings out the very nature of haptic as a term that is ultimately bounded with emotional grasping.

In that sense, a 'haptic space' can be described as a continuum of bodily and emotional response. Berenson [5] supports this notion by noting that our bodily response to the 'tactile properties' of a space highly depends on our understanding of their ability to affect and 'touch' us, while Fisher [6] addresses haptic as the merging of the bodily senses and the affective aspect of what creates them.

Drawing on the above, the first step towards creating the Strings installation started with the simple question of 'how to design a haptic space', a space, that is, whose qualities can trigger our senses and affect our bodily responses - and their embodied emotional aspect. Pallasmaa [7] and Bloomer and Moore [8] provide an answer to that 
question by writing respectively that such a space can be created "through $a$ strengthened sense of materiality, hapticity, texture" or "by generating sensations through textural change that link [the] space's haptic materiality with the bodies that inhabit it". The next question I had to take into consideration was 'how to incorporate the bidirectional relation of affect between bodily responses and the space that encompasses them within the spatial design itself'. In other words, how can I design an environment that is not only able of evoking bodily expressions but is also able of capturing them and 'feeding' them back to its 'organism'.

It was my intention to engender that bi-directional relation of affect strictly through the two agents of the interaction: the installation space and the body of the visitors, without having to embed external systems into their channel of communication. Consequently, I needed to establish a tangible form of interaction between the installation space and its visitors without using distant modes of interaction like sensors and vision-based tracking systems. To model such a form of intimate, tactile interaction, I focused my attention on the design of an innovative textile surface that would define the installation space, and that would itself be capable of 'perceiving' the bodily expressions of the users. This prototype fabric promotes a mode of interaction, which as Pallasmaa [9] puts it, springs from the tactile sensibility of "enhanced materiality, nearness and intimacy".

\section{THE INTERFACE}

\section{Design and Application}

The prototype consists of a non-conductive micro-tulle that has conductive wire strings (silver-plated copper, diameter of $0.10 \mathrm{~mm}$ ) embedded on both its outer sides, horizontally on the one and vertically on the other. Thus, the embedded strings form a grid whose conductive rows and columns do not make contact within the same plane unless they are compressed by touch. The conductive elements of the grid are wired up and connected to a computer allowing for the prototype to simulate a tactile, numerical interface that is activated by touch and whose resolution depends on the density of the conductive grid. 


\section{EVA 2009 London Conference 6-8 July

A

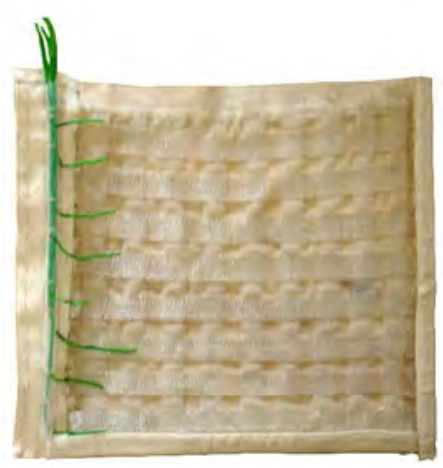

$\mathrm{C}$

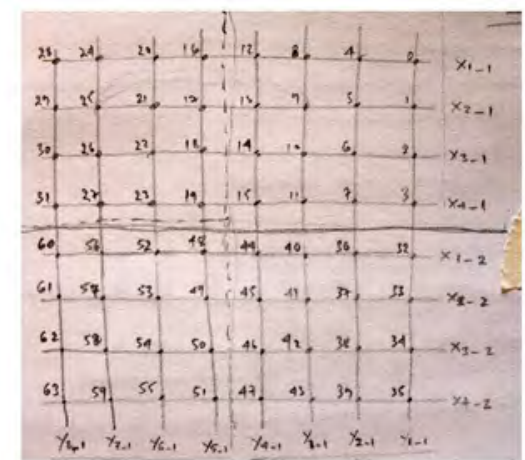

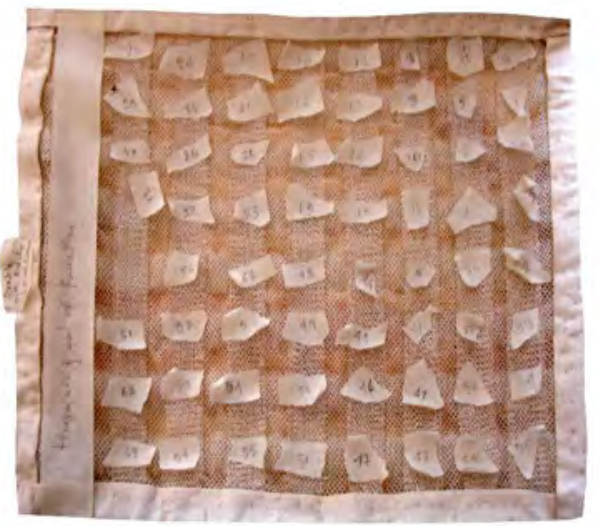

$\mathrm{B}$

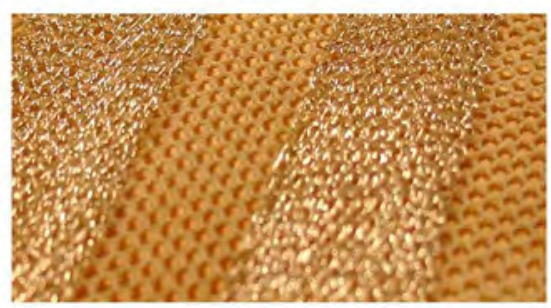

$\mathrm{D}$

Figure 2. Prototype sample $0.20 \times 0.20 \mathrm{~m}$.

A. Wired conductive elements, B. Conductive grid nodes, C. Grid nodes as matrix elements, D. Weaving detail.

My first approach was to connect the wired conductive strings to a complex of keypad encoders, which were in turn connected to an Arduino microcontroller. Each physical node of the conductive grid could, in this way, be perceived within the Arduino programming environment as an element of a matrix whose rows and columns are accordingly equivalent to the parallel and vertical conductive strings of the fabric. That would allow for the gestures of the users upon the interface to be captured as strings of compressed grid nodes, and eventually as strings of values that respond to the matrix elements. Although this solution worked well for small-scale testings, as the surface of the prototype was expanding and the number of the conductive strings was scaling up, the complexity of the circuitry and the coding suggested that another approach should be considered.

As a result, I decided to replace the keypad encoders with shift registers, a solution that drastically decreased the number of required components to half. 


\section{EVA 2009 London Conference 6-8 July

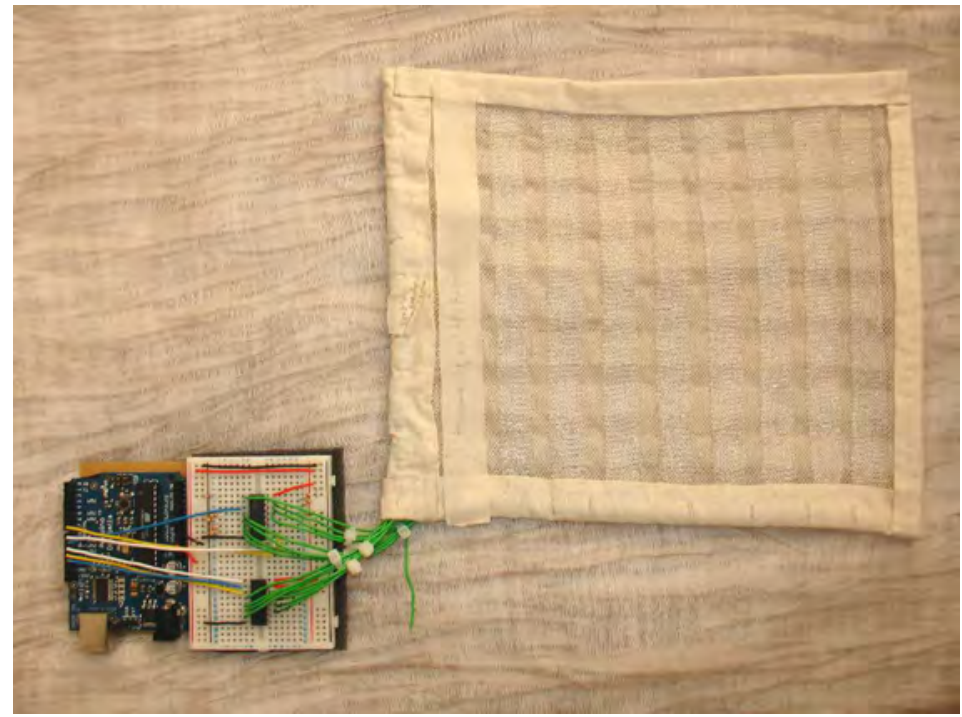

Figure $3.8 \times 8$ conductive strings grid, connected to shift registers.

As an approach it works almost identically to the fore-mentioned by capturing the gestures of the users upon the interface as arrays of compressed grid nodes, and 'transducing' them into strings of values that respond to the virtual matrix elements. However, it is much more elegant given the simplicity of scaling up it provides by daisy-chaining the necessary number of registers and slightly adjusting the code each time the grid expands. Moreover, this approach allows the 'grasping' of numerous simultaneous touched points upon the interface when more than one user is engaging with the interface. The touched points are then passed to MAX/MSP as strings of integers that respond to distinct gestures to generate sound, as I will shortly explain in more detail.

\section{Textural Qualities}

Both sides of the prototype are layered with a translucent fabric upon which a pattern I have developed after an extract from Italo Calvino's Invisible Cities and the relation of spatial perception with emotions is being printed. Selected areas of the printed fabric are then embroidered with a variety of different stitches and materials that vary in colour and weight. This process results into a highly textured surface that acts as the skin of the prototype. 


\section{EVA 2009 London Conference 6-8 July

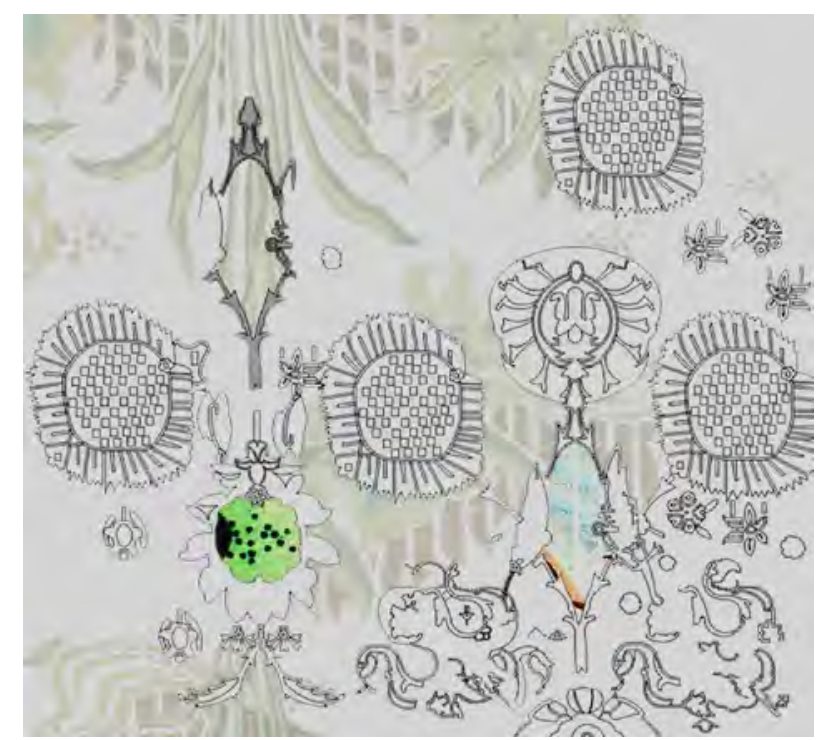

Figure 4. Print detail.

With the conductive grid acting as the 'nerves' of the prototype and the processed textile acting as its skin, a quite abstract representation of the spatial interface as a living organism evolves, and sets the ground for a bi-directional relation of affect between the envelope of the space and the bodies it encloses. The textured envelope attempts to intrigue the users' senses of vision and touch, aiming to evoke bodily engagement.

\section{Sound}

As soon as the users engage with the envelope through the medium of touch, their gestures are translated into sound. That enables a straightforward relation between the visual / haptic qualities of the interface and the generated sound, allowing for gestural patterns to be 'choreographed' and perceived both by the haptic qualities that engender them and by the audio output they generate. Different audio samples of stringed instruments map within MAX/MSP the different textural / chromatic qualities of the processed prototype skin; within each textural area, a central grid node is assigned a given sound, and acts as the 'command centre' for its peripheral nodes. 


\section{EVA 2009 London Conference 6-8 July

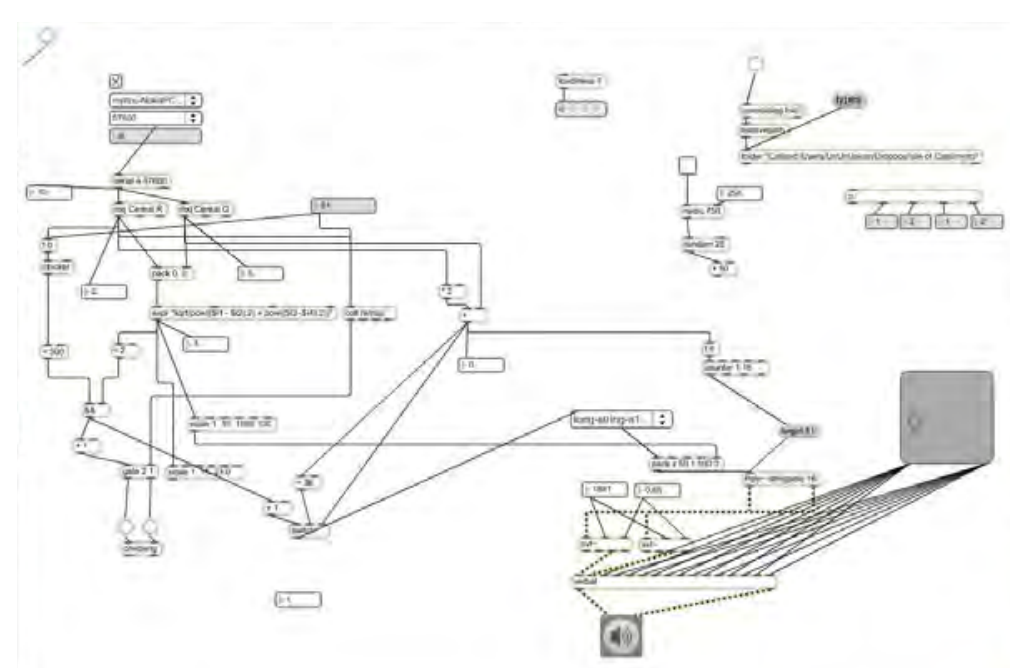

Figure 5. MAX/MSP patch.

Consequently, within a certain radius - defined by the size of each distinct textural area - the sound of all neighbouring nodes is interpolating with respect to their distance from the central node. As each node is perceived within MAX/MSP as a set of coordinates that respond to its position within the virtual matrix, the distance between two successive touched nodes can easily be calculated by applying the Pythagorean theorem on the matrix-corresponding X-Y coordinates of the two nodes. Because the sound that each triggered node generates is relational to the previously triggered node, the result of each gesture comes as an organic, non pre-determined sound composition. A time interval parameter determines whether two successive triggered nodes are part of a single gesture or not. When more than one person is engaging with the interface the sound is produced as the merged outcome of their embodied engagement with the interface and with each other.

When installed, the envelope can be approached from both its inner and outer side. As the weaving, print and embroidery of the envelope allows a certain level of translucency, the figures of the users become part of its patterns. Thus, apart from an auditory-oriented collaboration of the users' gestures, a visual level of interaction among them also affects the orchestration of their bodily expressions.

\section{CONCLUSION}

This paper presents my study on the design of Strings, an installation that attempts to model a bi-directional relation of affect between a responsive haptic space and the expressive bodies of its users. The installation is entirely constructed of a highly textured fabric prototype whose weaving involves conductive threads in a manner that they form a conductive grid. The crossovers of the grid-threads are within a programming environment perceived as elements of a matrix, enabling the prototype to act as a physical numerical interface. The bodily gestures of the users upon the prototype can therefore be captured in a tangible manner as a series of compressed grid 


\section{EVA 2009 London Conference 6-8 July \\ Myrto Karanika}

nodes, which are in turn 'transduced' into strings of matrix elements that correspond to the compressed nodes.

Each conductive grid node of the prototype is being assigned to an audio sample of a stringed instrument. As the colours and the variety of textures of the prototype affect the way the users engage with it, every single gesture of theirs upon the prototype generates different musical compositions of strings affecting the resonance and auditory output of the installation. A circular cause-and-effect interplay between user-and-space that is immediate, physical and tangible is this way engendered: the spatial qualities - texture and sound - 'orchestrate' the bodily gestures, while they are constantly being affected by the bodily expressions themselves.

Strings is an ongoing project that is still in an early stage of development. Having tested it yet only in a very small-scale, my next goal is to exhibit a $1.6 \times 0.7 \mathrm{~m}$ prototype, and to take into consideration a user engagement evaluation before proceeding with the final installation design. The description I have provided so far applies only to smallscale testing. Therefore, some of the application details may differ in the future. Apart from expected changes and improvements, the future development of Strings sees an additional feature that reverses the process described so far: using an algorithm that saves the generated sound output, I would like to be able to remap the gestures of the users upon the prototype through the medium of printing and embroidery.

\section{AKNOWLEDGEMENTS}

The work presented in this paper is being developed as part of my MFA in Computational Studio Arts degree. I would like to thank my tutors Janis Jefferies, Jane Prophet and Andrew Shoben. I would especially like to thank Alex Zivanovic for his support in coding and circuitry, and Jeremy Keanan and Olly Farshi for their input in the sound design and the recordings of the string samples.

\section{References}

[1] DELEUZE, G and GUATTARI, F. A thousand plateaus : capitalism and schizophrenia. London : Athlone, 1988.

[2] MERLEAU-PONTY, M. The primacy of perception and other essays on phenomenological psychology, the philosophy of art, history and politics. Evanston IL : Northwestern University Press, 1964.

[3] KANT, I. Critique of pure reason. London : Macmillan, 1990.

[4] PATERSON, M. The senses of touch : haptics, affects and technologies. Oxford : Berg, 2007.

[5] BERENSON, B. The Florentine painters of the renaissance. London : G.P. Putnam's Sons, 1906.

[6] FISHER, J. Relational sense : towards a haptic aesthetics. Parachute 87, 1, 1997. pp. 4-11.

[7] PALLASMAA, J. The eyes of the skin : architecture and the senses. London : Academy Editions, 2005.

[8] BLOOMER, K and MOORE, C. Body, memory and architecture. New Haven, CT : Yale University Press, 1978. 


\section{EVA 2009 London Conference 6-8 July \\ Myrto Karanika}

[9] PALLASMAA, J. Hapticity and time : notes on fragile architecture. Architectural Review, 207, 2000. pp.78-84. 